\title{
Die intracelluläre Lokalisation von Phenolasen im Tabakblatt
}

\section{Doctoral Thesis}

Author(s):

Hofer, Andreas

Publication date:

1964

Permanent link:

https://doi.org/10.3929/ethz-a-000122808

Rights / license:

In Copyright - Non-Commercial Use Permitted 
Diss ETH

\title{
Die intracelluläre Lokalisation von Phenolasen im Tabakblatt
}

\author{
Von der \\ Eidgenössischen Technischen \\ Hochschule in Zürich \\ zur Erlangung
}

der Würde eines Doktors der technischen Wissenschaften

genehmigte

Promotionsarbeit

Vorgelegt von

Andreas Hofer

dipl. Ing.-Agr.

von Arni bei Biglen, $\mathrm{BE}$

Referent: Herr Prof. Dr. A. Frey-Wysseing

Korreferent: Herr Prof. Dr. P. MAtiLe

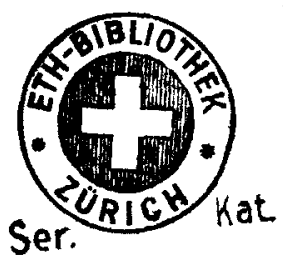

1964

Würzburg

Universitätsdruckerei H. Stürtz AG 
Ob reduzierte Chinone als Substrate der Phenolasen im Verlaufe der $\mathrm{CO}_{2}$-Assimilation eine Rolle spielen, muß dahingestellt bleiben; es ist weiter wohl kaum anzunehmen, daß Phenolasen eine Rolle in der photosynthetischen Phosphorylierung spielen.

Hess (1958) bringt die Phenolaseaktivität in einen direkten Zusammenhang mit dem Eiweißstoffwechsel. Tatsächlich steigt in Roggen (Szarkowski 1958) und Mais (Haskins 1955) während der Keimung die Phenolaseaktivität stark an; eine ursächliche direkte Beziehung ist jedoch nicht bewiesen.

Zusammenfassend sei betont, daß mit der vorliegenden Arbeit die Lokalisation der Phenolase, also eines Enzyms, dessen physiologische Rolle noch weitgehend ungeklärt ist, in Chloroplasten festgestellt wurde.

\section{Zusammenfassung}

1. Die Löslichkeit der Phenolasen erschwert eine einwandfreie quantitative Abgrenzung des Enzymanteils der Chloroplastensedimente von jenem des Grundplasmas im Überstand.

2. Aus Homogenaten von Tabakblättern in hypotonischen Medien (Wasser; 1/15 m Phosphatpuffer) sind 10\% der Katecholaseaktivität und ca. $40 \%$ der Chlorogensäureoxydase sedimentierbar (30 min $80000 \mathrm{~g}$ ); in hypertonischen Medien (0,6 m Mannit; 0,4 $\mathrm{m}$ Saccharose) betragen die entsprechenden Anteile $20 \%$ bzw. $60 \%$.

3. Die sedimentierbare Phenolaseaktivität läßt sich zu über $70 \%$ in den Chloroplasten nachweisen (fraktionierte Zentrifugation).

4. Durch wiederholtes Waschen der isolierten Chloroplasten werden die Phenolasen kontinuierlich freigesetzt. Nach der vierten Waschung läßt sich keine Aktivität mehr in den sedimentierbaren Chloroplastenfragmenten nachweisen.

5. Aus isolierten Chloroplasten können die Phenolasen durch Ultraschallbehandlung oder durch Suspension in hypotonischem Medium vollständig freigesetzt werden.

6. Elektronenmikroskopische Aufnahmen zeigen, daß der Phenolasegehalt der Chloroplasten mit dem Erhaltungszustand in engem $\mathrm{Zu}$ sammenhang steht. Gewaschene Chloroplasten bestehen nur mehr aus Grana- und Stromalamellen von unterschiedlicher Erhaltung.

7. Aus den genannten Resultaten wird geschlossen, daß die Phenolasen nicht strukturgebunden, sondern frei im Chloroplastenstroma vorliegen.

8. Die höchste Phenolaseaktivität wurde in Homogenaten von jungen wachsenden Blättern festgestellt.

9. Die Veränderung des Quotienten Katecholase-/Chlorogensäureoxydaseaktivität unter verschiedenen Versuchsbedingungen läßt vermuten, daß die Oxydation von Katechol und Chlorogensäure von zwei verschiedenen Enzymen katalysiert wird. 